\title{
Plasma samples from mouse strains and humans demonstrate differ- ent in vitro susceptibilities to complement activation
}

\author{
Barry W. Neun, ${ }^{1}$ Gábor Szénási, ${ }^{2,3,4,5}$ János Szebeni, ${ }^{2,3,4,5,6}$ and Marina A. Dobrovolskaia ${ }^{1 *}$ \\ ${ }^{I}$ Nanotechnology Characterization Laboratory, Frederick National Laboratory for Cancer Research sponsored \\ by the National Cancer Institute, Frederick, MD, USA** \\ ${ }^{2}$ Nanomedicine Research and Education Center, Institute of Pathophysiology, Semmelweis University, Buda- \\ pest, Hungary \\ ${ }^{3}$ Institute of Pathophysiology, Semmelweis University, Budapest, Hungary \\ ${ }^{5}$ SeroScience Ltd., Budapest, Hungary \\ ${ }^{4}$ Department of Nanobiotechnology and Regenerative Medicine, Faculty of Health, Miskolc University, Miskolc, \\ Hungary \\ ${ }^{5}$ Institute of Clinical Experimental Research, Semmelweis University, Budapest, Hungary \\ ${ }^{6}$ Institute for Translational Medicine, Medical School, University of Pécs, Hungary
}

Submitted: October 29, 2018

Accepted: November 19, 2018

Published: November 11, 2018

\section{Abstract}

Complement activation can be evaluated in vitro using plasma or serum from animals and human donors, and in vivo using animal models. Despite many years of research, there is no harmonized approach for the selection of matrix and animal models. Herein, we present an in vitro study investigating intra- and inter-species variability in the complement activation. We used the liposomal formulation of amphotericin, AmBisome, as a model particle to assess the magnitude of the complement activation in plasma derived from various mouse strains and individual human donors. We demonstrate that mouse strains differ in the magnitude of complement activation by liposomes and cobra venom factor in vitro. Inter-individual variability in complement activation by AmBisome and cobra venom factor was also observed when plasma from individual human donors was analyzed. Such variability in both mouse and human plasma could not be explained by the levels of complement regulatory factors $\mathrm{H}$ and $\mathrm{I}$. Moreover, even though mouse plasma was less sensitive to the complement activation by CVF than human plasma, it was equally sensitive to the activation by AmBisome. Our study demonstrates the importance of mouse strain selection for in vitro complement activation analysis. It also shows that traditional positive controls, such as cobra venom factor, are not predictive of the degree of complement activation by nanomedicines. The study also suggests that besides complement inhibitory factors, other elements contribute to the inter- and intraspecies variability in complement activation by nanomedicines.

Keywords:

Hypersensitivity, infusion reactions, cobra venom factor, anaphylatoxins, CARPA, AmBisome, nanoparticles, in vitro, preclinical

\section{Abbreviations:}

- CVF: cobra venom factor

\footnotetext{
* Corresponding authors: Marina A. Dobrovolskaia, Nanotechnology Characterization Laboratory, Frederick National Laboratory for Cancer Research sponsored by the National Cancer Institute, Frederick, MD, USA E-mail: marina@mail.nih.gov and Janos Szebeni, Nanomedicine Research and Education Center, Semmelweis University, 1089 Nagyvárad tér 4, Budapest, Hungary jiszebeni2@gmail.com

** The content of this publication does not necessarily reflect the views or policies of the Department of Health and Human Services, nor does mention of trade names, commercial products, or organizations imply endorsement by the U.S. Government.
} 
- CARPA: complement activation-related pseudoallergy

- CFH and CFI: complement inhibitory factors

- Th-1 and Th-2: Subsets of T lymphocytes expressing CD4 on their surface

\section{Rationale and purpose}

Herein we describe an in vitro study conducted to answer a question about inter- and intra-species variability in complement activation by traditional agonist, cobra venom factor (CVF) and model nanoformulation, liposomal amphotericin (AmBisome). We wanted to know if all mouse strains are equally responsive to AmBisome with complement activation. We also assessed human donor plasma for potential inter-individual variability in the complement activation by CVF and AmBisome. Lastly, we compared mouse and human in vitro response to both agonists to understand which matrix represents a more sensitive in vitro model. This information is important to inform preclinical safety studies of nanomedicines.

\section{Introduction}

A group of proteins produced by the liver and present in the bloodstream form socalled complement system ${ }^{1-3}$. The proteins in this system function to complement humoral and cellular immunity in detecting and eliminating invading pathogens, thus providing the name for the system ${ }^{1-3}$. Recent studies demonstrated that in addition to the innate immunity, complement activation plays an important role in regulating the adaptive immune response, and contributing to vaccine efficacy ${ }^{4,5}$. Undesirable activation of the complement system, however, may occur in response to certain drug products including but not limited to therapeutic proteins, nucleic acids, and nanotechnology-based formulations ${ }^{6-9}$. When such activation occurs, it results in the generation of so-called anaphylatoxins (e.g., complement split products $\mathrm{C} 3 \mathrm{a}, \mathrm{C} 4 \mathrm{a}$, and $\mathrm{C} 5 \mathrm{a}$ ) which trigger cardiopulmonary changes resembling type I hypersensitivity reactions $^{6-9}$. The true type I reactions involve drug-specific IgE. Since complement activation mediated anaphylaxis does not involve $\operatorname{IgE}$, this reaction is also called complement activation-related pseudoallergy or CARPA ${ }^{7,9}$. Even though CARPA is not specific to nanomedicines and occurs in response to other types of therapeutic products, it creates a particular hurdle for nanoformulations due to the complexity of their structure, composition, as well as the regulatory approval process. CARPA phenomenon and underlying mechanisms for nanomaterials have been studied extensively in the past decade. Despite these efforts, many unanswered questions still exist, and the research to cover existing gaps in understanding the complement contribution to infusion reactions is actively progressing ${ }^{8-10}$. Understanding the propensity of a test nanomaterial to activate the complement system is, therefore, very important both for preclinical safety and mechanism of action (MOA) studies. It is also recommended by international standard development organization such as, for example, ISO and ASTM International ${ }^{11,12}$.

Despite many years of research, there is still no harmonized in vitro assay and in vivo model to test for complement activation $^{13-15}$. Many popular in vitro methods rely on treating human or animal serum or plasma with a test-nanomaterial, followed by the evaluation of the treated samples for the presence of complement split products (C3a, C4a, C5a) or terminal complex $(\mathrm{sC} 5 \mathrm{~b}-9)^{13,15}$. Other commonly used in vitro assays include complement consumption $(\mathrm{CH}-50)^{14}$. Many nuances exist with regards to the type of matrix (serum vs. plasma), end-point of the complement activation (e.g., split product, terminal complex or consumption) and anticoagulants. While several laboratories agreed that hirudin is more complement friendly than other commonly used anticoagulants ${ }^{16-18}$, it is not broadly available to research labs. There is also no general consensus, regarding the type and source of matrix and end-points. Each known in vitro method has advantages 
and limitations. The same is true about animal models. Rodents (mice and rats), dogs and non-human primates are traditionally used to assess complement activation in vivo $^{7,19}$. Another valuable but not broadly used model is a pig $^{20,21}$. Discussions about the advantages and limitations of the in vivo models have also been extensively discussed $^{8,9,20,22}$.

\section{Materials and Methods}

Reagents

AmBisome was obtained from NIH Pharmacy (Bethesda, MD, USA). Human $\mathrm{iC} 3 \mathrm{~b}$ ELISA kit and CVF were purchased from Quidel (San Diego, CA, USA). Vacutainers with hirudin were purchased from Roche (Budapest, Hungary). Mouse CFH, CFI and $\mathrm{C} 3 \mathrm{a}$ kits as well as human $\mathrm{CFH}$ and CFI kits were from MyBiosource (San Diego, CA, USA).

\section{Animals}

Blood was drawn from several mouse strains commonly used in preclinical research. These strains included Balb/c, CD1, $\mathrm{C} 3 \mathrm{H} / \mathrm{HeN}, \mathrm{C} 57 \mathrm{BL} / 6$ and DBA1. The blood was drawn into tubes containing the hirudin as anticoagulant; the collection was performed by Bioreclamation Inc. (Westbury, NY, USA).

Research Donor Blood

Human blood specimens were collected from ten healthy donor volunteers under NCI at Frederick Protocol OH99-C-N046. Plasma was prepared by centrifugation and stored at a nominal temperature of $-20^{\circ} \mathrm{C}$ prior to use in the complement activation in vitro study or for analysis of inhibitory factors concentrations.

\section{Complement Assays}

Mouse plasma from various strains or human plasma from ten donor volunteers was mixed at 4:1 volume ratio with controls or liposomes, incubated for 30 minutes at $37{ }^{\circ} \mathrm{C}$ and analyzed by ELISA for the presence of the complement split products. PBS was used as the negative control, CVF $(10 \mathrm{U} / \mathrm{mL})$ was used as the positive control. AmBisome was tested directly from stock to mimic condition relevant to the infusion of this drug in vivo. Levels of complement inhibitory factors ( $\mathrm{CFH}$ and $\mathrm{CFI}$ ) in the same mouse and human plasma specimens as those used for the complement activation experiments were assessed using commercial kits and according to the manufacturer's instructions.

\section{Statistical analysis}

Data analysis and plotting were performed using GraphPad Prism7 for Windows (GraphPad Software, La Jolla, California, USA).

\section{Results}

The magnitude of in vitro complement activation by AmBisome varies between mouse strains.

In this part of our study, we wanted to assess potential intra-species variability in the complement activation. We hypothesized that, despite being inbred, various mouse strains might have different sensitivity to the complement activation by liposomes. We chose five strains (Balb/c, CD-1, $\mathrm{C} 3 \mathrm{H} / \mathrm{HeN}, \mathrm{C} 57 \mathrm{BL} / 6$, and DBA1) which are commonly used in preclinical research and have known differences in their immune responses. For example, Balb/c and CD-1 mice are known to be Th-2 biased animals and, therefore, are preferred for studies investigating the sensitization potential of test-substances ${ }^{23}$. C57BL/6 mice are known to be Th-1 biased animals, and therefore, they are preferred species for the studies of vaccines and autoimmunity ${ }^{23}$. $\mathrm{C} 3 \mathrm{H} / \mathrm{HeN}$ and DBA-1 animals do not have a known Th1/Th2 bias. However, they are commonly used in the innate immunity studies $^{23}$. When treated in vitro with controls and AmBisome, plasma from these animals demonstrated various levels of the complement activation as evidenced by the presence of C3a (Figure 1A).

To allow comparison between the strains, we determined stimulation index (SI). The SI is the ratio between complement split products in the test sample and that in the 
negative control. We calculated and compared SI to both the positive control (CVF) and AmBisome. The highest degree of the complement activation was observed in plasma from Balb/c and CD-1 mice (Figure
1B). Plasma from C57BL/6 mice demonstrated the least response to CVF and was completely insensitive to AmBisome (Figure 1B). Plasma from two other strains demonstrated a moderate response to both treatments (Figure 1B).

A
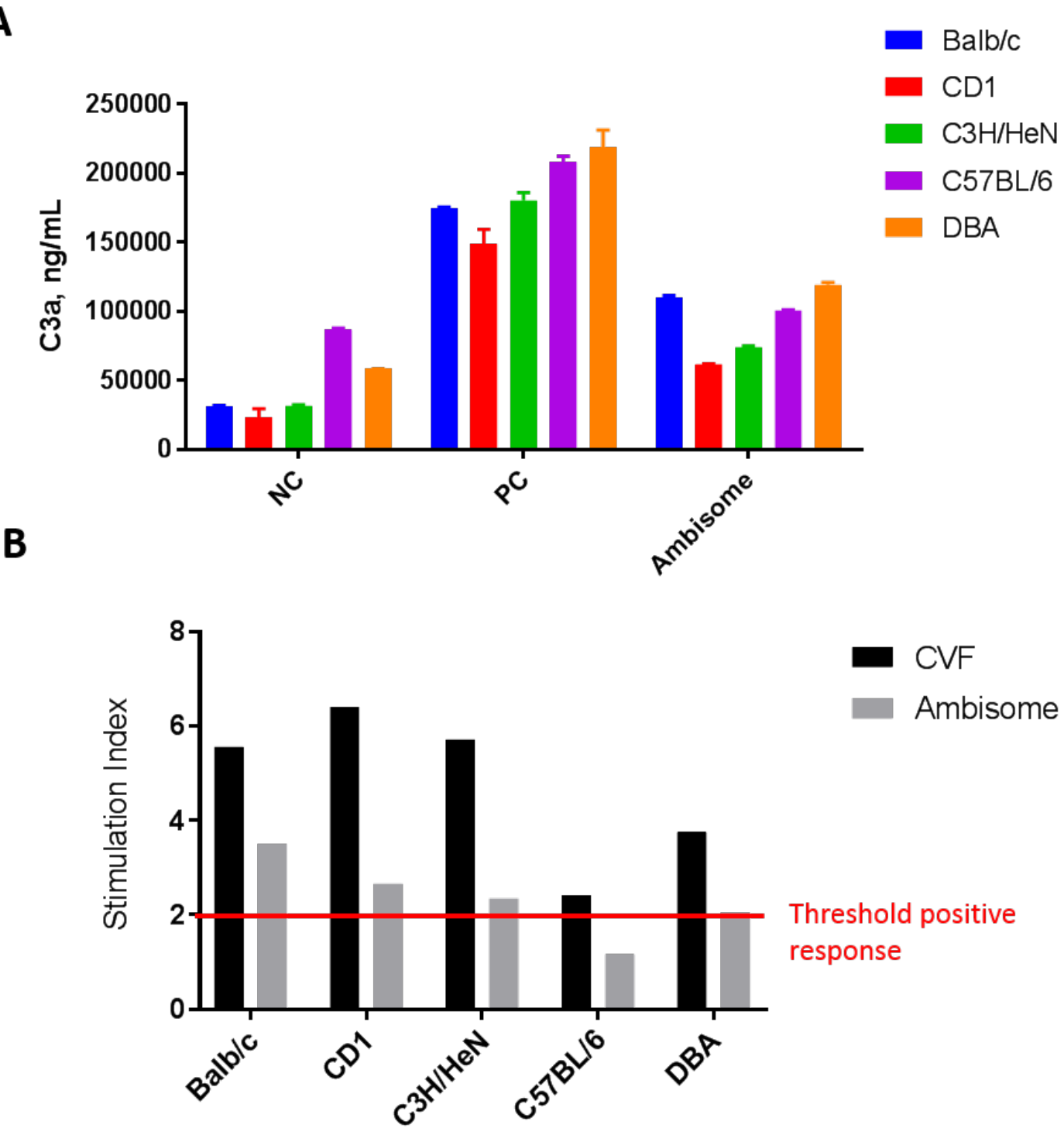

Figure 1. In vitro analysis of Complement activation by AmBisome in mouse plasma. Plasma specimens were derived from inbred animals of various strains. Complement activation by negative control (NC), positive control (PC) and AmBisome was assessed in vitro using the procedure described in materials and methods. PBS and CVF were used as NC and PC, respectively. (A) Levels of C3a in plasma 30 minutes after treatment with controls and AmBisome. Each bar shows the mean and standard deviation (N=3). (B) Stimulation index was calculated for the positive control (CVF) and AmBisome for each mouse strain by dividing the mean C3a concentration in the test sample by that in the negative control. The red line shows the physiologically significant threshold of the positive response $(\geq 2$-fold $)$. 
Mouse plasma concentrations of $\mathrm{CFH}$ and CFI do not explain inter-strain variability in complement activation

To get an insight into the role of the complement regulatory factors as a potential reason for differences in the complement activation observed among tested mouse strains, we analyzed concentrations of factors $\mathrm{H}$ and I (CFH and CFI, respectively) in the same plasma sets used for the complement activation study. We found that levels of these factors vary drastically between Balb/c, CD-1, C3H/HeN, C57BL/6 and DBA1 (Figure 2). Interestingly, the levels of these factors were in reverse relationship in that strains with high levels of CFH (e.g.,
Balb/c and C57BL/6) had low plasma concentration of CFI (Figure 2). Likewise, strains with low concentrations of $\mathrm{CFH}$ (e.g., $\mathrm{C} 3 \mathrm{H} / \mathrm{HeN}$ and $\mathrm{DBA}$ ) had high, relative to other strains, concentrations of CFI (Figure 2). Among tested strains, CFI concentration was the highest in plasma from CD-1 mice, in which CFH levels were also relatively high (Figure 2). Concentrations of either complement inhibitory factor per se could not explain the differences in the magnitude of the complement activation by Doxil or CVF in plasma from individual mouse strain (Figure 2, compare heat map of $\mathrm{CFH}$, CFI vs. SI by $\mathrm{CVF}$ and AmBisome).

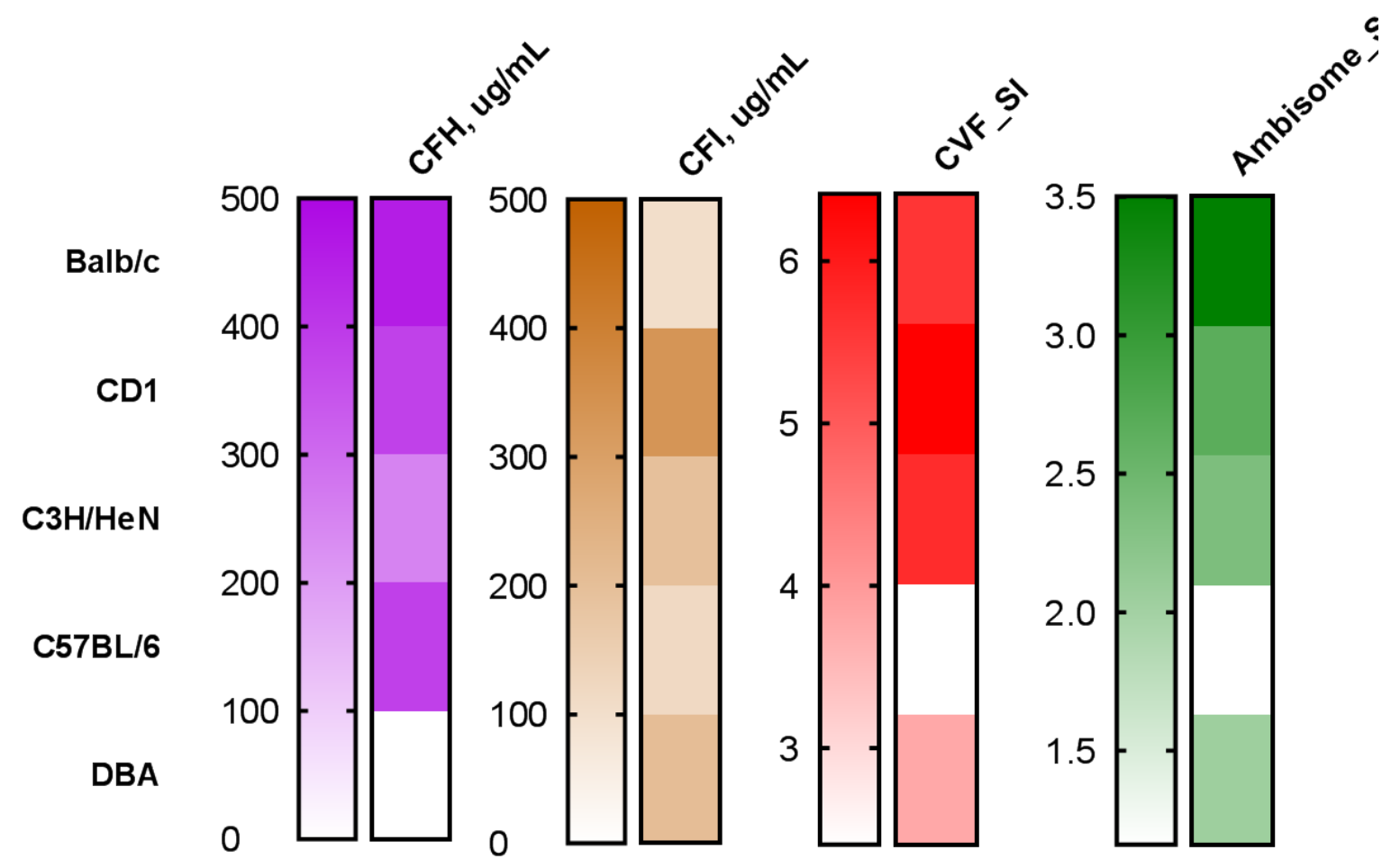

Figure 2. Levels of complement inhibitory factors and stimulation indices in plasma from various mouse strains. Plasma from individual mouse strains was analyzed by commercial ELISA for the presence of complement inhibitory factors H and I (CFH and CFI, respectively). The data is plotted in a heatmap format, where the darker color corresponds to the higher concentration. The same plasma samples were used for the analysis of the complement activation. The stimulation indices (SI) presented in Figure 1B, are plotted here in the heatmap format to contrast to CFH and CFI concentrations. The darker color in the SI heatmaps also corresponds to the higher complement activation than lighter colors.

The magnitude of in vitro complement activation by AmBisome varies between individual human donors

To understand the relevance of our in vitro study using mouse plasma to the preclinical in vitro complement activation assay typically conducted in the plasma of human donors, we treated plasma from ten healthy 
donor volunteers with PBS, CVF or AmBisome and measured levels of the complement split product iC3b (Figure 3A). Similar to the study in plasma from various mouse strains, we observed inter-individual variability in the complement activation by both CVF and AmBisome (Figure 3A). Interestingly, comparison of the SI showed that, unlike the mouse, human plasma is extremely sensitive to CVF as evident by SIs above 50 (Figure 3B). This data is in contrast to that observed in mouse plasma wherein SIs to CVF were between 2 and 6 (Figure 1B). Stimulation indices of AmBisome in human plasma were comparable to that observed in mouse plasma (compare SI AmBisome in Figure 1B and Figure 3B; in both matrices, SI-AmBisome varies between 2 and 4).

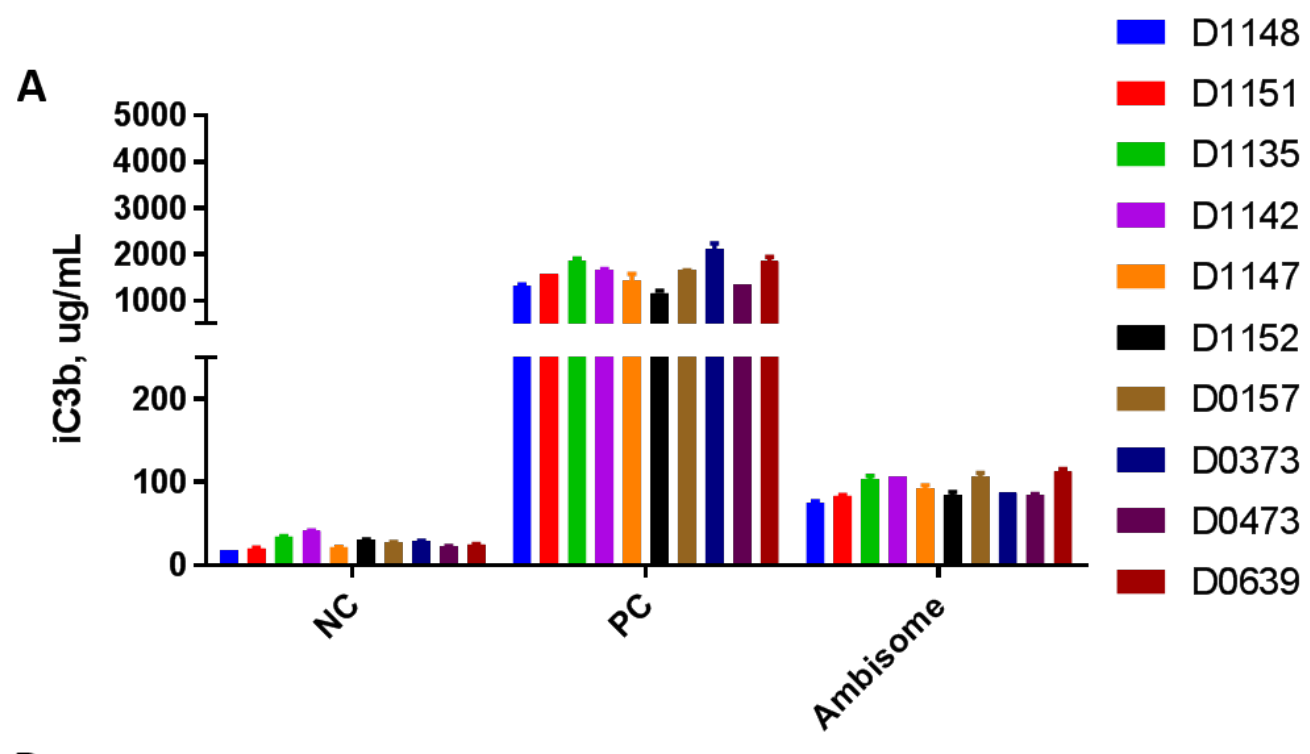

B

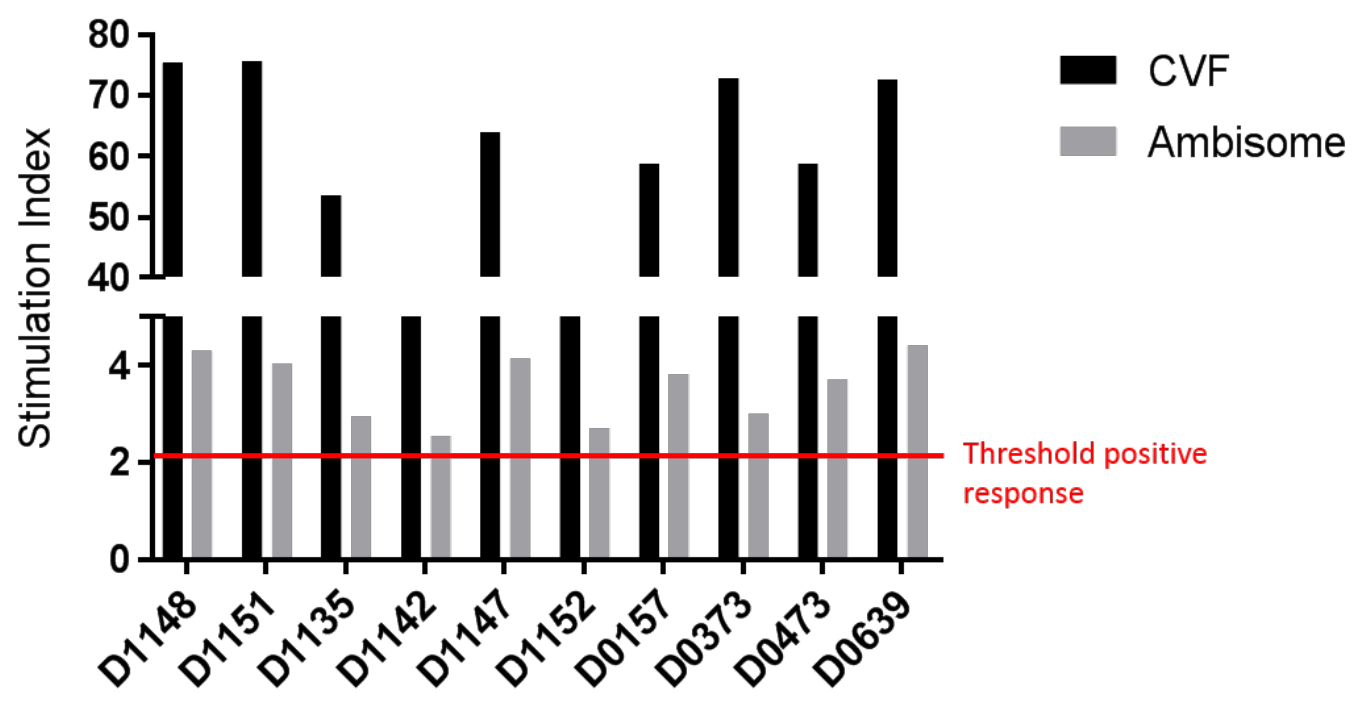

Figure 3. In vitro complement activation by AmBisome in human plasma. Plasma from ten healthy donor volunteers denoted as letter $D$ followed by a number was studied in vitro as described in materials and methods. Levels of complement split product $i C 3 b$ were measured by ELISA. (A) Levels of iC3b in individual plasmas after treatment with negative control (NC), positive control (PC) and AmBisome. Each bar shows the mean and standard deviation (N=3). PBS and CVF were used as a positive and negative control, respectively. (B) Stimulation index was calculated by dividing the iC3b levels in CVF or AmBisome by that in the negative control of the individual donor. Red line shows the physiologically relevant threshold of the positive response (i.e., at least 2-fold above the baseline) 
Human plasma concentrations of $\mathrm{CFH}$ and CFI do not explain inter-individual variability in complement activation

To understand whether variability in the complement activation by AmBisome is due to the different levels of expression of the complement inhibitory factors in plasma of individual donors, we measured the levels of these factors in the same plasma samples as those used for the complement activation study. We found that while $\mathrm{CFH}$ and CFI levels vary between individuals (Figure 4). However, the concentration of these factors per se cannot explain the variability observed in the complement activation study (Figure 4 compare $\mathrm{CFH}$ and CFI heatmap to AmBisome-SI). The further statistical analysis did not reveal a direct correlation between $\mathrm{CFH}$ or
CHI concentrations and AmBisome stimulation indices (data not shown). This finding is in agreement with our earlier study comparing complement SI in plasma from different donors treated in vitro with Doxil (a PEGylated liposomal doxorubicin) and CFH/CFI levels ${ }^{24}$. In our current study, we used plasma specimens from some donors which were also used in our previous project investigating Doxil-mediated complement activation ${ }^{24}$. We also included Doxil as a nanoparticle-relevant positive control. Even though the plasma from these donors was drawn at a different time, the Doxil SIs in the current study (data not shown) matched those reported by us ear$\operatorname{lier}^{24}$. Similar to the earlier findings, AmBisome SIs did not match CVF SIs in plasma from individual donors (Figure 4).

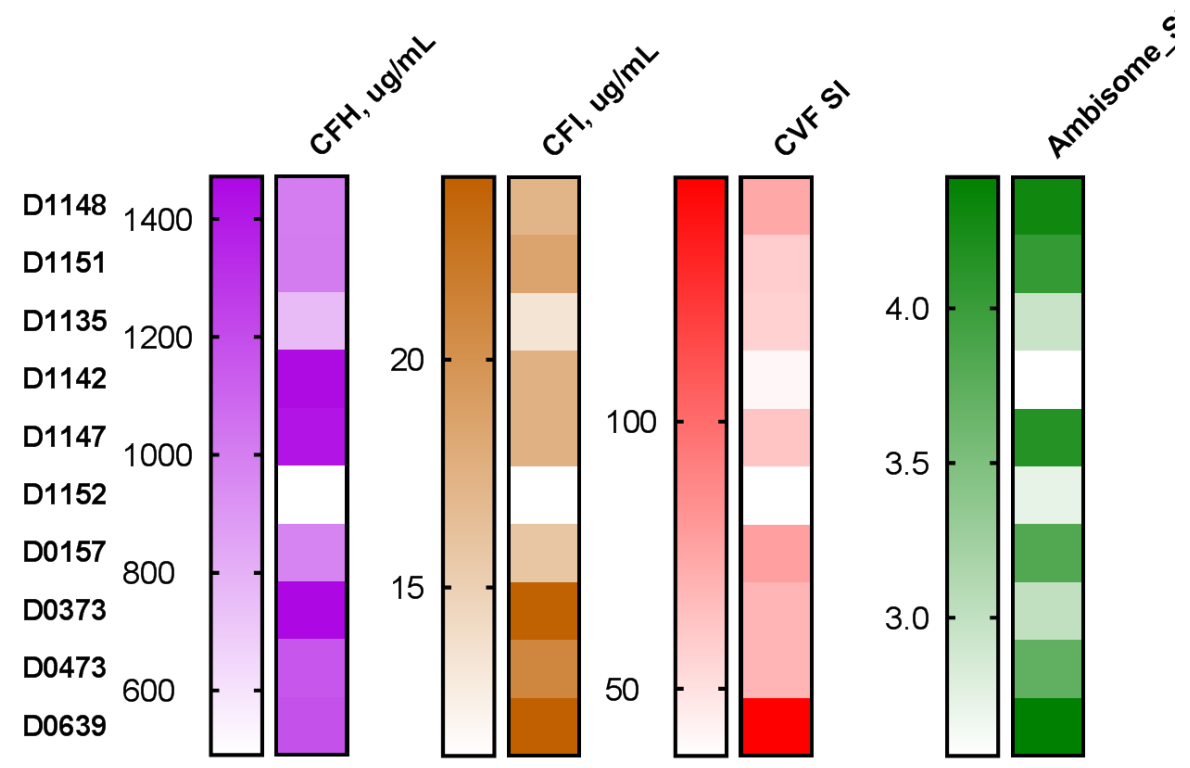

Figure 4. Levels of complement inhibitory factors and stimulation indices in plasma from individual human donors. Commercial ELISA was used to analyze plasma from ten human donors for the presence of complement inhibitory factors $H$ and I (CFH and CFI, respectively). The data is plotted in a heatmap format, where the darker color corresponds to the higher concentration. The same plasma samples were used for the analysis of the complement activation. The stimulation indices (SI) presented in Figure 3B, are plotted here in the heatmap format to contrast to CFH and CFI concentrations. The darker color in the SI heatmaps also corresponds to the higher complement activation than lighter colors. Statistical analysis did not reveal a direct correlation between CFH or CFI concentrations and AmBisome SIs.

\section{Discussion}

The findings described herein inform the field of preclinical characterization of nanomaterials. The data demonstrate, that if the mouse matrix is used for in vitro analy- sis of complement activation by nanomaterials, Balb/c or CD-1 mice appear to be the most optimal source of the matrix due to their higher sensitivity to the complement activation by both CVF and liposomes (Figure 1 and 2). Likewise, our data suggest 
that $\mathrm{C} 57 \mathrm{BL} / 6$ mice are not an appropriate source of plasma for in vitro complement activation studies due to their minimal response to both the control and the liposome stimuli. Our data further suggest that in a situation where a difference in the in vitro evaluation of the complement activation by liposomes is observed among published studies, one has to take a closer look at the mouse strain used to source the plasma as a potential source of discrepancy in the in vitro test results. Inter-individual variability in the complement activation by AmBisome in vitro was not surprising and it lines up with clinical observations that not all donors develop complement-activation mediated pseudoallergy reaction in response to AmBisome ${ }^{25}$. Interestingly, we found that even though mouse plasma was less sensitive than human plasma to the complementtriggering activity of the CVF, it showed comparable sensitivity to the complement activation by AmBisome (Figure 1B and 3B). This data suggest that complement activation by $\mathrm{CVF}$ and AmBisome involve different pathways. Activation of complement may occur through different pathways. At least three such pathways have been described and include classical, lectin and alternative pathways ${ }^{26,27}$. All of them converge on the $\mathrm{C} 3$ component of the complement. Therefore, if a screening is performed with the goal to identify a specific pathway, the agonist of that pathway is a preferable positive control. However, when the aim is simply to identify a propensity of a test-material to activate the complement system, inclusion of any agonist which results in a sufficient generation of the $\mathrm{C} 3$ split product is generally acceptable. Examples of commercially available positive controls include bet-glucan zymosan A, $\mathrm{CVF}$ and heat activated gamma globulins. In our study we analyzed C3 split products as the indication of the complement activation and used CVF as a traditional control. Despite generating high levels of the C3 split products in both mouse and human plasma, this control turned out to be unreliable to estimate complement activation by nanomedicines. Therefore, our data suggest that using a traditional agonist (e.g., CVF) for the estimation of matrix suitability to screen complement activating activity of nanomedicines may be misleading. The important finding of our study is that in vitro assays used to estimate the magnitude of complement activation by nanomedicines should be validated using the nanomedicine in question. We also found that AmBisome SIs do not match Doxil SIs in the plasma of the same individuals (data not shown). Collectively, this data is in line with earlier studies by Benasutti et al., demonstrating that complement opsonization rates observed with one nanomedicine do not accurately predict complement binding to other structurally similar nanoparticles ${ }^{22}$. Similar to our findings in mouse plasma (Figure 2), individual levels of $\mathrm{CFH}$ and $\mathrm{CFI}$ varied in plasma of human donors (Figure 4) and per se could not explain the magnitude of the complement activation by AmBisome. This the finding is in agreement with an earlier study demonstrating the lack of direct correlation between $\mathrm{CFH} / \mathrm{CFI}$ levels and the magnitude of the complement activation by Doxil $^{24}$.

\section{Summary}

Collectively, the results of this in vitro study deliver several take-home messages to the research community dealing with the preclinical characterization of nanotechnology-based products. First, the source of the matrix is important. Even though the human matrix is preferable, when it is not available, it can be substituted with mouse plasma. However, close attention has to be paid to the mouse strain from which the plasma is obtained. Second, complement activation by liposomes can be influenced by many factors including but not limited to the levels of the complement regulatory factors. Third, traditional controls such as CVF and other nanomedicines, even those belonging to the same class of nanocarriers (e.g., liposomes in our studies), 
cannot be used to predict the magnitude of the complement activation by the given nanoformulation. Fourth, in vitro complement assays should be validated using the nanomedicine in question as opposed to relying simply on the traditional positive control, especially when such positive control activates complement through the mechanism different from that triggered by the nanomedicine in question".

Acknowledgment

This work was supported by the European Union ("NanoAthero" project FP7-NMP-2012 LARGE-6-309820) and the Applied Materials and Nanotechnology Center of Excellence, Miskolc University, Miskolc, Hungary. The study was supported in part (B.W.N and M.A.D) by federal funds from the National Cancer Institute, National Institutes of Health, under contract HHSN261200800001E.

\section{Authors' contributions}

M.A.D and J.S. conceived the study, planned experiments, analyzed data and wrote the manuscript. B.W.N. performed experiments. G.S. contributed to the experiment planning and data analysis.

\section{Conflict of Interests}

The authors declare no conflicts of interest. For signed statements, please contact the journal office: editor@precisionnanomedicine.com

Quote as: Barry W, Neun BW, Szénási G, Szebeni J, Dobrovolskaia MA, Plasma samples from mouse strains and humans demonstrate different susceptibilities to complement activation, Precis. Nanomed. 2018;1(3):208-217, https://doi.org/10.33218/prnano1(3).181029.2

\section{References}

1 Holers, V. M. Complement and its receptors: new insights into human disease. Annu Rev Immunol. 32 433-459, doi:10.1146/annurev-immunol-032713-120154, (2014).

2 Nonaka, M. Evolution of the complement system. Subcell Biochem. 80 31-43, doi:10.1007/978-94-017-8881-6_3, (2014).

3 Tambourgi, D. V. \& van den Berg, C. W. Animal venoms/toxins and the complement system. Mol Immunol. 61 (2), 153-162, doi:10.1016/j.molimm.2014.06.020, (2014).

4 Reddy, S. T. et al. Exploiting lymphatic transport and complement activation in nanoparticle vaccines. Nat Biotechnol. 25 (10), 1159-1164, doi:10.1038/nbt1332, (2007).

5 Thomas, S. N. et al. Engineering complement activation on polypropylene sulfide vaccine nanoparticles. Biomaterials. 32 (8), 2194-2203, doi:10.1016/j.biomaterials.2010.11.037, (2011).

6 Bedocs, P. et al. Hypersensitivity reactions to intravenous lipid emulsion in swine: relevance for lipid resuscitation studies. Anesth Analg. 119 (5), 1094-1101, doi:10.1213/ane.0000000000000396, (2014).

7 Szebeni, J. Complement activation-related pseudoallergy: a stress reaction in blood triggered by nanomedicines and biologicals. Mol Immunol. 61 (2), 163-173, doi:10.1016/j.molimm.2014.06.038, (2014).

8 Szebeni, J. Mechanism of nanoparticle-induced hypersensitivity in pigs: complement or not complement? Drug Discov Today. 23 (3), 487-492, doi:10.1016/j.drudis.2018.01.025, (2018).

9 Szebeni, J. et al. Hypersensitivity to intravenous iron: classification, terminology, mechanisms and management. Br J Pharmacol. 172 (21), 5025-5036, doi:10.1111/bph.13268, (2015). 
10 Moghimi, S. M. Nanomedicine safety in preclinical and clinical development: focus on idiosyncratic injection/infusion reactions. Drug Discov Today. 23 (5), 1034-1042, doi:10.1016/j.drudis.2017.11.006, (2018).

11 ASTM_International. (2018).

12 Organization, I. S. (2017).

13 Neun, B. W., Ilinskaya, A. N. \& Dobrovolskaia, M. A. Analysis of Complement Activation by Nanoparticles. Methods Mol Biol. 1682 149-160, doi:10.1007/978-1-4939-73521_13, (2018).

$1 \overline{4}$ Pham, C. T. et al. Application of a hemolysis assay for analysis of complement activation by perfluorocarbon nanoparticles. Nanomedicine. 10 (3), 651-660, doi:10.1016/j.nano.2013.10.012, (2014).

15 Dobrovolskaia, M. A. Pre-clinical immunotoxicity studies of nanotechnology-formulated drugs: Challenges, considerations, and strategy. J Control Release. 220 (Pt B), 571-583, doi:10.1016/j.jconrel.2015.08.056, (2015).

16 Bexborn, F. et al. Hirudin versus heparin for use in whole blood in vitro biocompatibility models. J Biomed Mater Res A. 89 (4), 951-959, doi:10.1002/jbm.a.32034, (2009).

17 Cedrone, E. et al. Anticoagulants Influence the Performance of In vitro Assays Intended for Characterization of Nanotechnology-Based Formulations. Molecules. 23 (1), doi:10.3390/molecules23010012, (2017).

18 Kopp, R. et al. Effect of hirudin versus heparin on hemocompatibility of blood contacting biomaterials: an in vitro study. Int J Artif Organs. 28 (12), 1272-1277 (2005).

19 Moghimi, S. M., Wibroe, P. P., Szebeni, J. \& Hunter, A. C. Surfactant-mediated complement activation in beagle dogs. Int Immunopharmacol. 17 (1), 33-34, doi:10.1016/j.intimp.2013.05.012, (2013).

20 Dezsi, L. et al. Features of complement activation-related pseudoallergy to liposomes with different surface charge and PEGylation: comparison of the porcine and rat responses. $\mathrm{J}$ Control Release. 195 2-10, doi:10.1016/j.jconrel.2014.08.009, (2014).

21 Fulop, T. et al. Complement activation in vitro and reactogenicity of low-molecular weight dextran-coated SPIONs in the pig CARPA model: Correlation with physicochemical features and clinical information. J Control Release. 270 268-274, doi:10.1016/j.jconrel.2017.11.043, (2018).

22 Benasutti, H. et al. Variability of Complement Response toward Preclinical and Clinical Nanocarriers in the General Population. Bioconjug Chem. 28 (11), 2747-2755, doi:10.1021/acs.bioconjchem.7b00496, (2017).

23 Watanabe, H., Numata, K., Ito, T., Takagi, K. \& Matsukawa, A. Innate immune response in Th1- and Th2-dominant mouse strains. Shock. 22 (5), 460-466 (2004).

24 Neun, B. W., Barenholz, Y., Szebeni, J. \& Dobrovolskaia, M. A. Understanding the Role of Anti-PEG Antibodies in the Complement Activation by Doxil in vitro. Molecules. 23 (7), doi:10.3390/molecules23071700, (2018).

25 Szebeni, J. Complement activation-related pseudoallergy: a new class of drug-induced acute immune toxicity. Toxicology. 216 (2-3), 106-121, doi:10.1016/j.tox.2005.07.023, (2005).

26 Bajic, G., Degn, S. E., Thiel, S. \& Andersen, G. R. Complement activation, regulation, and molecular basis for complement-related diseases. Embo j. 34 (22), 2735-2757, doi:10.15252/embj.201591881, (2015).

27 Noris, M. \& Remuzzi, G. Overview of complement activation and regulation. Semin Nephrol. 33 (6), 479-492, doi:10.1016/j.semnephrol.2013.08.001, (2013). 